\title{
A Comparative Study of Different Doses of Lignocaine on Prevention of Etomidate Induced Myoclonus during Induction of Anesthesia
}

\author{
Sarat Chandra Jayasingh ${ }^{1} \quad$ Sikata Nanda ${ }^{2}$ Ramakanta Mohanty ${ }^{3}$ Sidharth S. Routray ${ }^{4} \quad$ Issan C. Dalai ${ }^{4}$ \\ ${ }^{1}$ Department of Surgical Gastroenterology, Srirama Chandra Bhanja \\ Medical College, Cuttack, Odisha, India \\ 2 Department of Community Medicine, Srirama Chandra Bhanja \\ Medical College Cuttack, Odisha, India \\ ${ }^{3}$ Department of Surgery, Fakir Mohan Medical College, Balasore, \\ Odisha, India \\ ${ }^{4}$ Department of Anesthesiology and Critical Care, Srirama Chandra \\ Bhanja Medical College Cuttack, Cuttack, Odisha, India Anesthesiology and Critical Care, Srirama Chandra Bhanja Medical College Cuttack, Odisha 753007, India \\ Address for correspondence Sidharth S. Routray, MD, Department of \\ (e-mail: drsidharth74@gmail.com). \\ Int J Recent Surg Med Sci 2022;8:84-88.
}

\begin{abstract}
Background and Aims Etomidate, an intravenous (IV) induction agent known for its stable myocardial action, can produce myoclonus which can be detrimental for the cardiac patients. Though lignocaine has proven its efficacy in attenuating the etomidate-induced myoclonus, the ideal dose of lignocaine is not known. The aim of our trial was to analyze two different doses of lignocaine on the occurrence and intensity of etomidate induced myoclonus.

Materials and Methods A total of 120 patients were randomly assigned into three groups of 40 each. Patients in group A were injected lignocaine $0.5 \mathrm{mg} / \mathrm{kg}$ intravenously, group-B patients were injected lignocaine $1 \mathrm{mg} / \mathrm{kg}$ intravenously, and group-C patients were injected saline placebo. After 2 minutes, anesthesia was induced with $0.3 \mathrm{mg} / \mathrm{kg}$ of etomidate over 30 seconds. The patients were assessed for myoclonus using clinical severity scoring system during first 2 minutes of induction. Our primary outcome was the incidence of myoclonus. The severity of myoclonus and adverse effects were the secondary outcomes.

Results No remarkable variation was found regarding demographic profile among

Keywords

- anesthesia

- etomidate

- lignocaine

- myoclonus

- induction three groups. Incidence of myoclonus in groups A and B was 35\% and in group C was $98 \%$, the difference being statistically significant. Both doses of lignocaine reduced the severity of myoclonus up to same extent.

Conclusion Pretreatment with IV lignocaine $0.5 \mathrm{mg} / \mathrm{kg}$ and $1 \mathrm{mg} / \mathrm{kg}$ IV remarkably decreased the occurrence and severity of myoclonus induced by etomidate up to same extent.
\end{abstract}

DOI https://doi.org/ 10.1055/s-0041-1740503. ISSN 2455-7420.

\footnotetext{
(c) 2022. Medical and Surgical Update Society. All rights reserved. This is an open access article published by Thieme under the terms of the Creative Commons Attribution-NonDerivative-NonCommercial-License, permitting copying and reproduction so long as the original work is given appropriate credit. Contents may not be used for commercial purposes, or adapted, remixed, transformed or built upon. (https://creativecommons.org/ licenses/by-nc-nd/4.0/)

Thieme Medical and Scientific Publishers Pvt. Ltd., A-12, 2nd Floor, Sector 2, Noida-201301 UP, India
} 


\section{Introduction}

An imidazole derivative, etomidate, is one of the rapidly acting intravenous (IV) inducing agent commonly used for induction in cardiac patients posted for surgery because of its cardiovascular stability. IV etomidate of $0.3 \mathrm{mg} / \mathrm{kg}$ does not produce any cardiac instability but it can produce side effects like injection site pain, phlebitis, adrenal suppression, and myoclonus, as well as emergence agitation, nausea, and vomiting. But myoclonus itself can produce serious complications in cardiac patients. ${ }^{1}$ However, the mechanism of this myoclonus after etomidate induction is not clear yet. As in recent years, fat emulsion of etomidate has replaced etomidate-in-water adverse effects, such as phlebitis, and pain on injection was reduced but it had no impact on incidence of myoclonus. ${ }^{2}$ Myoclonus is defined as sudden jerks of involuntary muscles for a short period. In literature, the incidence of myoclonus has been recorded maximum up to $85 \%{ }^{3}$ Myoclonus can produce dangerous complication in patients having open global injury, with full stomach and patients with cardiac instability, as it increases intraocular pressure, aspiration of gastric contents, and exacerbates cardiac instability in these patients, respectively. Many drugs like midazolam, opioids, dexmedetomidine, and lignocaine have been tried to reduce the occurrence of myoclonus induced by etomidate but each one has its own limitations. ${ }^{4}$ Though few studies have confirmed the role of lignocaine in managing etomidate-induced myoclonus, there is still no unified understanding about the dose selection of lignocaine. ${ }^{5}$ Lignocaine in a dose of $(1-1.5 \mathrm{mg} / \mathrm{kg})$ is usually used for attenuation of sympathetic stress response to endotracheal intubation which can be an added advantage. There is paucity of studies in literature regarding efficacy of ideal dose of lignocaine on the occurrence and severity of myoclonus. This trial was conducted to find out the efficacy of two different doses of IV lignocaine on the occurrence and severity of myoclonus induced by etomidate.

\section{Materials and Methods}

This trial was conducted after obtaining institutional ethical committee clearance and written informed consent from patients in a tertiary care hospital. A total of 140 cases posted for different cardiac surgery under general anesthesia from August 2019 to July 2020 were selected. Patients with allergy to lignocaine on sedative or opioids within 24 hour before surgery, patients having adrenocortical suppression, neurological, and psychiatric disorders were excluded from the study. A total of 120 patients of American Society of Anesthesiologists grade I/II were randomly divided by computerized randomization into A, B, and C groups, each with 40 patients. Randomization was done using a computer-generated random number list. The allocation sequence was consecutively numbered and was kept in a sealed opaque envelope. Anesthesiologist, not involved in the study, opened the envelope in operation theater and prepared the study drug accordingly. After patients arrived in the operation theater, preoperative pulse rate, oxygen saturation, invasive blood pressure, central venous pressure, and electrocardiography were monitored. IV access was established using a 20 -gauge IV cannula and ringer lactate was started. Anesthesia induction protocol of group A was done with lignocaine $0.5 \mathrm{mg} / \mathrm{kg}$ + etomidate $0.3 \mathrm{mg} / \mathrm{kg}$, anesthesia induction protocol of group B was done with lidocaine $1 \mathrm{mg} / \mathrm{kg}+$ etomidate $0.3 \mathrm{mg} / \mathrm{kg}$, and anesthesia induction protocol of group $\mathrm{C}$ was done with normal saline + etomidate $0.3 \mathrm{mg} / \mathrm{kg}$. Study drug was given 2 minutes before induction.

Anesthesiologist, unaware of the study drug injection, observed the etomidate-induced myoclonus and any other adverse effects for 2 minutes. Grading of myoclonus was done as follows: 0 , no myoclonus; 1 , mild (finger or wrist); 2 , moderate (face and leg); 3 , severe (fast abduction of any limb). Assisted ventilation was done with $100 \%$ oxygen during myoclonus. After the 2 minutes of observation, vecuronium bromide of $0.1 \mathrm{mg} / \mathrm{kg}$, midazolam of $0.05 \mathrm{mg} / \mathrm{kg}$, and fentanyl of $2 \mu \mathrm{g} / \mathrm{kg}$ were administered to all the three groups for tracheal intubation. Then anesthesia was maintained with sevoflurane 1.5 to $2 \%$ with oxygen and air.

The primary aim of our study was to assess the occurrence of myoclonus during the first 2 minutes following the injection of etomidate. The secondary aim was to evaluate the effect of study drug on intensity of myoclonus induced by etomidate and to find out any other adverse effects.

We have calculated the sample size, taking the incidence of etomidate-induced myoclonus as primary aim. Based on the findings of a previous study, ${ }^{5}$ the sample size was calculated taking incidence of myoclonus in placebo group estimated to be $80 \%$. For $50 \%$ reduction in the incidence of myoclonus, by taking $\alpha=0.05$ and $\beta=0.2$ and taking $15 \%$ dropout rate, minimum number of patients required in each group is 40 .

\section{Statistical Methods}

Statistical calculations were done using the Graphpad Prism 7.0 (GraphPad Software Inc., San Diego, CA). Normal distribution of the continuous data was given as mean \pm standard deviation and analyzed using the Student's $t$-test. Nonnormally distributed data were analyzed using the MannWhitney $U$-test. Categorical data were analyzed using Chisquare test or Fisher's exact test. A $p$-value of $<0.05$ was considered as statistically significant.

\section{Results}

Out of 140 patients judged for eligibility, 20 patients were excluded for not meeting the inclusion criteria. A total 120 patients who met inclusion criteria were divided into three groups of 40 patients each and were analyzed (-Fig. 1). There were no remarkable variations in the demographic characteristics among the three groups ( - Table 1 ). Pretreatment with lignocaine before etomidate induction resulted in remarkable decrease in occurrence of myoclonus in groups $\mathrm{A}$ and $B$ compared with group $C$ which was statistically significant (35\% in group A, 35\% in group B and 98\% in group C; - Table 2; - Fig. 2). Besides, lignocaine also decreased the 


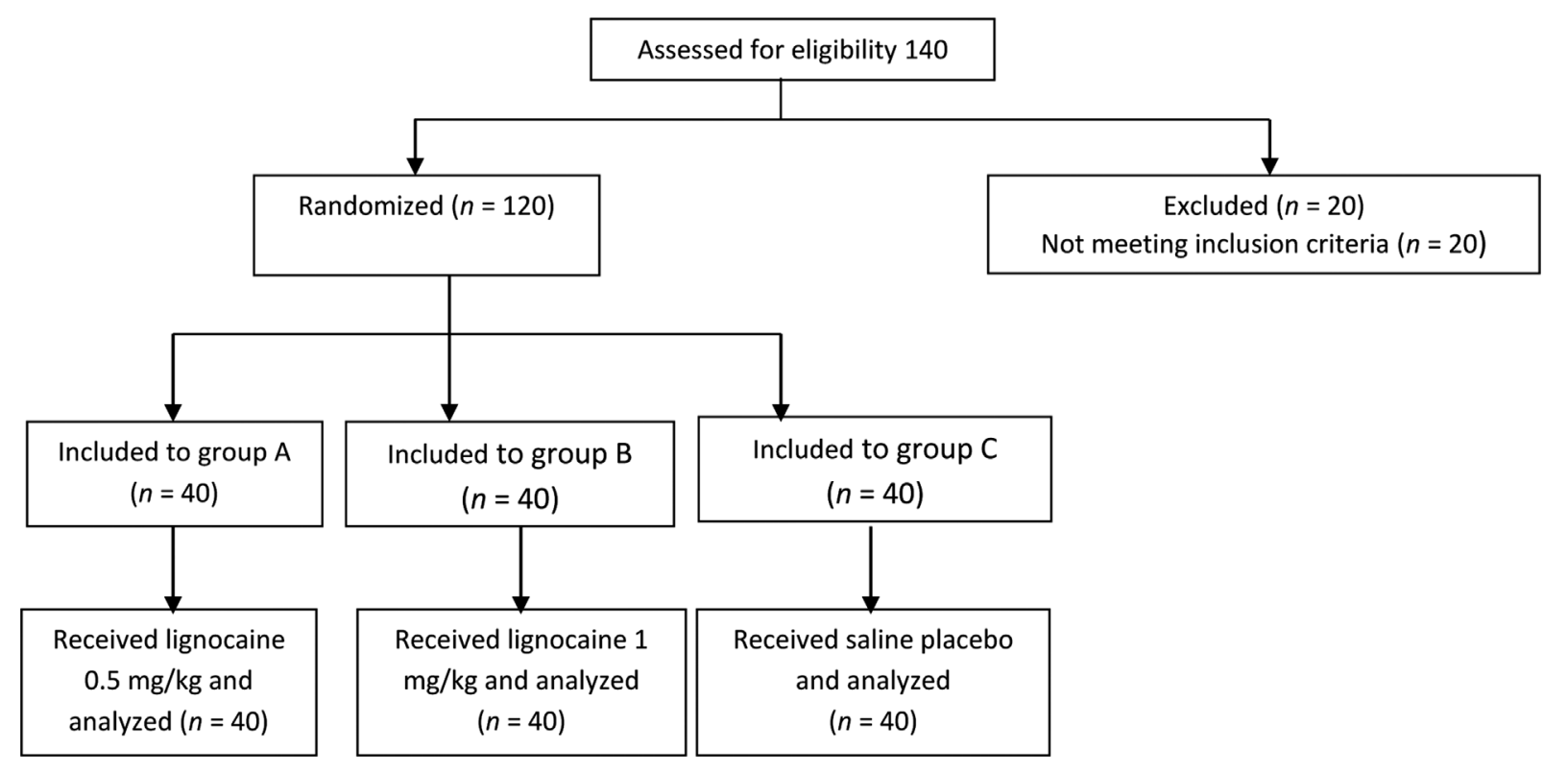

Fig. 1 Consort flow diagram.

Table 1 Demographic characteristics

\begin{tabular}{|l|l|l|l|l|}
\hline Variables & Group A & Group B & Group C & $p$-Value \\
\hline Age $(\mathrm{y})$ & $45.4 \pm 10.8$ & $42.7 \pm 9.8$ & $43.1 \pm 10.7$ & 0.534 \\
\hline Sex (M:F) & $30: 10$ & $32: 8$ & $28: 12$ & 0.215 \\
\hline Weight $(\mathrm{kg})$ & $60.35 \pm 8.1$ & $58.8 \pm 9.1$ & $59.0 \pm 10.2$ & 0.612 \\
\hline ASA status (I/II) & $35: 5$ & $34: 6$ & $36: 4$ & 0.594 \\
\hline
\end{tabular}

Abbreviations: F, female; $M$, male.

Table 2 Incidence of myoclonus

\begin{tabular}{|l|l|l|l|}
\hline Incidence of myoclonus & Group A & Group B & Group C \\
\hline No of patients (\%) & $14(35)$ & $14(35)$ & $39(98)$ \\
\hline
\end{tabular}

intensity of myoclonus in both groups A and B when compared with group $C$ which was statistically significant (-Figs. 3 and $\mathbf{4}$ ). There were no significant variations in the occurrence of any other side effects among the three groups.

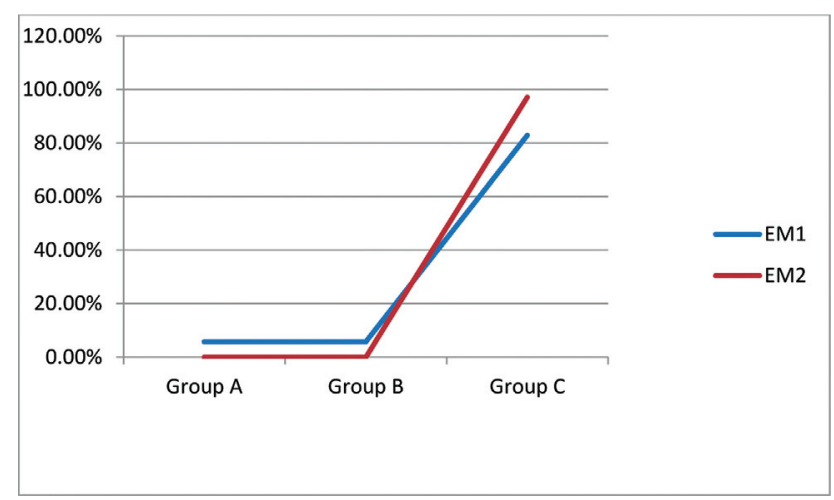

Fig. 2 Lignocaine response on incidence of myoclonus on 1 minute (EM1) and 2 minutes (EM2).

\section{Discussion}

Current study has shown that both doses of IV lignocaine when injected prior to etomidate decreased the occurrence and intensity of etomidate induced myoclonus. Etomidate is a type of short-acting and nonbarbiturate IV anesthetic. It is extensively used in the induction of general anesthesia in cardiac patients. It has the advantages like minimal effect on

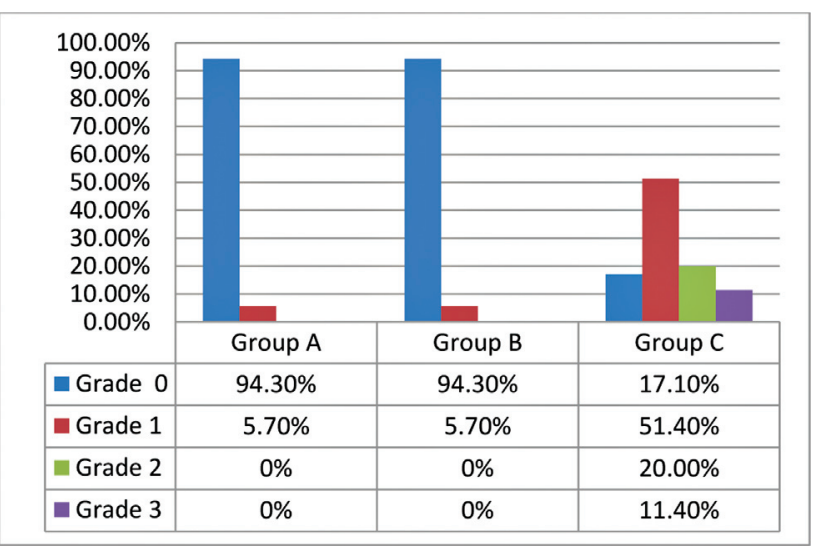

Fig. 3 Effects of lignocaine on myoclonus at 1 minute of etomidate administration. 


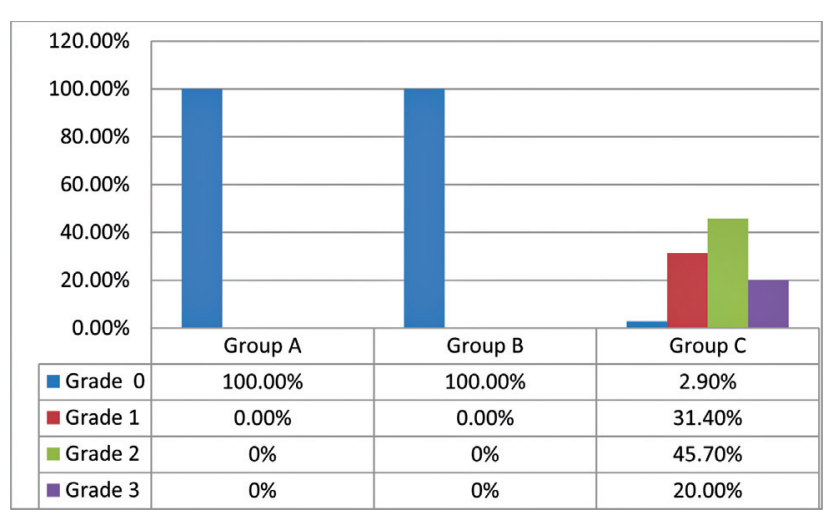

Fig. 4 Effects of lignocaine on myoclonus at 2 minutes of etomidate administration.

circulation state and hemodynamic, and it is especially suitable for patients with unstable cardiovascular system. However, using etomidate for anesthesia induction will increase the incidence of adrenal suppression, injection pain, phlebitis, and myoclonus, as well as emergence agitation, nausea, vomiting, and other side effects. ${ }^{6}$ Related studies have reported that when etomidate is used for anesthesia induction, the incidence of myoclonus reaches 50 to $80 \%$. The occurrence of myoclonus during anesthesia induction will affect the stability of hemodynamic and also increase the risk of emergence agitation and other adverse reactions. ${ }^{7}$ Therefore, various methods to prevent etomidate induced myoclonus has been the focus of clinical research. The mechanisms of etomidate-induced myoclonus are complex and not completely clear, and currently known mechanisms are as follows: (1) neural inhibitory pathway is more easily inhibited than excitatory pathway, and etomidate inhibits brainstem reticular formation function through gamma aminobutyric acid (GABA) receptor, increases the sensitivity of neural pathway that controls skeletal muscle, and thus leads to increased neural discharge and myoclonus; (2) etomidate can be combined with dopamine receptor in brain striatum, substantia nigra and other structures, and thus inhibit the biological function of dopamine which is manifested as endogenous dopamine deficiency, that is, myoclonus. ${ }^{8}$ Many drugs, like dexmedetomidine, midazolam, fentanyl, and lignocaine, have shown their efficacy in reducing the occurrence of etomidate-induced myoclonus. ${ }^{9}$ Literature have shown that lignocaine can be an ideal pretreatment for prevention of myoclonus as it has a short, rapid onset of action, and least effect on cardiorespiratory system. Also, it blunts the sympathoadrenal response due to intubation and has no impact on recovery of anesthesia. ${ }^{10}$ All the studies in literature have used only low doses of IV lignocaine $(<0.5 \mathrm{mg} / \mathrm{kg})$ which has shown to remarkably reduce the occurrence of myoclonus due to etomidate. ${ }^{11}$ Lang et al in a meta-analysis concluded that the pretreatment with lignocaine reduced the incidence and severity of myoclonus due to etomidate with limited effect on hemodymamics. They suggested for further studies to validate that Singh et al in their study showed that $20 \mathrm{mg}$ of $2 \%$ lignocaine injection showed $56 \%$ reduction in the incidence of myoclonus. ${ }^{12}$ Similar results were observed in the study by Gultop et al. ${ }^{13}$ Injection of $2 \%$ lignocaine $(1 \mathrm{~mL})$ and saline was administered 30 seconds before induction with etomidate, and found that occurrence of myoclonus in the lignocaine group was $56.6 \%$ in comparison with $83 \%$ in the control group. However, the mechanism by which lignocaine prevents myoclonus is not clear. ${ }^{14}$

Reduction of the central nervous system excitability may be a possible explanation for myoclonus prevention. ${ }^{15}$ Different studies by Chen et al, ${ }^{16}$ and Xiao et al $^{17}$ concluded that lignocaine can reduce the etomidate induced myoclonus but were unable to opine about ideal dose of lignocaine. Yang ${ }^{18}$ in his study analyzed the effect of different dose of IV lignocaine on etomidate induced myoclonus and concluded that $1 \mathrm{mg} / \mathrm{kg}$ of IV lignocaine was better than $1.5 \mathrm{mg} / \mathrm{kg}$ of IV lignocaine in attenuating etomidate-induced myoclonus. Gupta and Gupta ${ }^{19}$ in their study also compared three different doses of lignocaine and concluded that low dose $(20 \mathrm{mg})$ of lignocaine was better than 40-mg lignocaine due to lack of side effects but both attenuated etomidate induced myoclonus up to same extent which was in agreement with our study.

\section{Conclusion}

Both doses of lignocaine reduced the incidence and severity of myoclonus up to same extent and are equally effective in controlling etomidate induced myoclonus.

\section{Conflict of Interest}

None declared.

\section{References}

1 Forman SA. Clinical and molecular pharmacology of etomidate. Anesthesiology 2011;114(03):695-707

2 Nyman Y, Von Hofsten K, Palm C, Eksborg S, Lönnqvist PA. Etomidate-Lipuro is associated with considerably less injection pain in children compared with propofol with added lidocaine. $\mathrm{Br}$ J Anaesth 2006;97(04):536-539

3 Chan CM, Mitchell AL, Shorr AF. Etomidate is associated with mortality and adrenal insufficiency in sepsis: a meta-analysis*. Crit Care Med 2012;40(11):2945-2953

4 Bergen JM, Smith DC. A review of etomidate for rapid sequence intubation in the emergency department. J Emerg Med 1997;15 (02):221-230

5 Zhao X, Bao R, Zhu J, et al. Pretreatment with butorphanol reduces myoclonus after etomidate. J Anesthesiol Clin Sci 2013;2(01):2

6 Van Keulen SG, Burton JH. Myoclonus associated with etomidate for ED procedural sedation and analgesia. Am J Emerg Med 2003; 21(07):556-558

7 Lang B, Zhang L, Li F, Lin Y, Zhang W, Yang C. Comparison of the efficacy and safety of remifentanil versus different pharmacological approaches on prevention of etomidate-induced myoclonus: a meta-analysis of randomized controlled trials. Drug Des Devel Ther 2019;13:1593-1607

8 Doenicke AW, Roizen MF, Kugler J, Kroll H, Foss J, Ostwald P. Reducing myoclonus after etomidate. Anesthesiology 1999;90 (01):113-119

9 Un B, Ceyhan D, Yelken B. Prevention of etomidate-related myoclonus in anesthetic induction by pretreatment with magnesium.J Res Med Sci 2011;16(11):1490-1494

10 Lang B, Zhang L, Yang C, Lin Y, Zhang W, Li F. Pretreatment with lidocaine reduces both incidence and severity of etomidate-induced 
myoclonus: a meta-analysis of randomized controlled trials. Drug Des Devel Ther 2018;12:3311-3319

11 Li YC, Liu G. [The effect of lidocaine pretreatment on etomidateinduced myoclonus]. Guide of China Medicine. 2011;9(34): 127-128

12 Singh KA, Gupta Ruchi, Aujla K Singh, et al. Efficacy of lignocaine versus midazolam in controlling etomidate-induced myoclonus: A randomized placebo-controlled study. Ains Shams Journal of Anesthesiology 2014;7(03):460-464

13 Gultop F, Akkaya T, Bedirli N, Gumus H. Lidocaine pretreatment reduces the frequency and severity of myoclonus induced by etomidate. J Anesth 2010;24(02):300-302

14 Voss LJ, Sleigh JW, Barnard JP, Kirsch HE. The howling cortex: seizures and general anesthetic drugs. Anesth Analg 2008;107 (05):1689-1703

15 Herrera-Peco I, Wix-Ramos R, Domínguez-Gadea L, et al. Changes in cerebral perfusion induced by etomidate in patients with temporal lobe epilepsy [in Spanish]. Rev Neurol 2009;49(11): 561-565

16 Chen Y, Chen HR, Xie LB, Xu XB, Li J, Liu YQ. The effect of lidocaine on etomidate-induced myoclonus by etomidate during the induction of general anesthesia. Beijing Med 2011;33(08): 642

17 Xiao D, Cheng ZG, Kong GY, Pan BB, Liu JS. Prevention of pretreatment with Lidocaine on myoclonus during anesthesia induction with Etomidate. China Journal of Modern Medicine 2011;21(23): 2915-2917

18 Yang XL. Effect of different doses of lidocaine on etomidateinduced myoclonus during general anesthesia induction. Hainan Yixueyuan Xuebao 2016;22(07):92-96

19 Gupta P, Gupta M. Comparison of different doses of intravenous lignocaine on etomidate-induced myoclonus: a prospective randomised and placebo-controlled study. Indian J Anaesth 2018;62 (02):121-126 